\title{
Efficacy of PD-1 Inhibitors in Older Non-small Cell Lung Cancer Patients
}

\author{
YUKIKO NAKAMURA ${ }^{1}$, KAZUHITO MIYAZAKI $^{1}$, NAOTO AIKO $^{1}$, YUKI MISUMI $^{1}$, YOKO AGEMI $^{1}$, \\ YURI TANIGUCHI ${ }^{1}$, MARI ISHI ${ }^{1}$, TSUNEO SHIMOKAWA ${ }^{1}$, HIROAKI OKAMOTO ${ }^{1}$ and TAKEHARU YAMANAKA ${ }^{2}$ \\ ${ }^{1}$ Department of Respiratory Medicine, Yokohama Municipal Citizen's Hospital, Yokohama, Japan; \\ ${ }^{2}$ Department of Biostatistics, Yokohama City University School of Medicine, Yokohama, Japan
}

\begin{abstract}
Background/Aim: We assessed the efficacy of immune checkpoint inhibitors (ICIs) in older patients because of the limited information regarding these patients. Patients and Methods: We retrospectively analyzed 66 consecutive patients $\geq 70$ years old with advanced non-small cell lung cancer (NSCLC). A total of 31 patients received ICIs (ICIs group) and 35 patients received only cytotoxic regimens (No ICIs group). Results: Patients with squamous cell carcinoma who received ICIs had better overall survival (OS) than those who did not (9.7 versus 4.7 months, $p=0.027)$. In multivariate analysis, treatment with ICIs [hazard ratio $(H R)=0.54,95 \%$ confidence interval $(C I)=0.30-0.98, p=0.044]$, good performance status ( $H R=0.30,95 \% C I=0.16-0.57, p=0.0003)$, and histology other than squamous cell carcinoma $(H R=0.41,95 \% C I=0.19-0.83$, $p=0.014)$ were significantly favorable factors for OS. Conclusion: ICIs may be effective for older patients with NSCLC, especially squamous cell carcinoma patients.
\end{abstract}

Immune checkpoint inhibitors (ICIs) have led to a remarkable improvement in prognosis in non-small cell lung cancer (NSCLC). On the basis of several clinical trials, the antiprogrammed death-1 (PD-1) antibodies nivolumab and pembrolizumab have been approved for the treatment of advanced NSCLC patients who have received previous cytotoxic chemotherapy (1-3). However, the median age of patients in these randomized clinical trials was early 60 s, and the cut-off age for subgroup analysis was 65 years old. However, the incidence of lung cancer is higher in the older population, and the median age at diagnosis of lung cancer is about 70 years (4).

This article is freely accessible online.

Correspondence to: Yukiko Nakamura, 56 Okazawa-cho, Hodogaya-ku, Yokohama, Kanagawa 240-8555, Japan. Fax: +81 453325599, Tel: +81 453311961, e-mail: y-tanji@fc4.so-net.ne.jp

Key Words: Older patients, non-small cell lung cancer, immune checkpoint inhibitor, anti PD-1 inhibitor, overall survival.
An analysis of patients who received nivolumab and pembrolizumab in real clinical settings showed that the median age at PD-1 inhibitor initiation was 69 years (interquartile range=69-75 years) (5). Recently, few studied reported the efficacy and safety of ICIs in older NSCLC patients. A metaanalysis in NSCLC patients showed similar efficacy of ICIs in both younger ( $<65$ years old) and older ( $\geq 65$ years old) patients. However, among NSCLC patients aged over 75, no prolonged overall survival (OS) was observed (6). Checkmate 153 reported data on the assessment of nivolumab in advanced NSCLC patients from a large predominantly community-based study. In this study, the median OS in patients aged 70 years or older was 10.3 months, and clinical benefit was observed (7). In an Italian cohort with advanced squamous cell carcinoma, it was suggested that the benefit from nivolumab and the tolerability in older patients were similar to that in the overall population (8).

However, information whether ICIs demonstrate a survival benefit compared with cytotoxic agents in older patients is limited, because of underrepresentation of this patient population in prospective clinical studies. We conducted this retrospective study to evaluate the efficacy of ICIs in older patients.

\section{Patients and Methods}

Patient selection. We retrospectively collected data from the medical records of consecutive patients $\geq 70$ years old diagnosed with NSCLC at the Yokohama Municipal Citizen's Hospital between January 2011 and March 2018. Of these patients, we included only patients previously treated with one or two chemotherapy regimens. Patients treated with ICIs as first-line therapy were excluded. Epidermal growth factor receptor (EGFR) tyrosine kinase inhibitor (TKI) treatment was not counted as a regimen. We excluded patients treated with ICIs as fourth or later lines for the purpose of evaluating older patients in a similar setting to that of previous prospective studies of NSCLC (1-3).

We divided them into patients treated with only cytotoxic regimens without ICIs (No ICIs group) and those treated with ICIs as second- or third-line therapy (ICIs group).

Evaluation and statistical analyses. All categorical variables were analyzed by the chi-square test or Fisher's exact test. Progression- 
Table I. Characteristics of patients.

\begin{tabular}{|c|c|c|c|c|}
\hline Characteristics & All & No ICIs $(\mathrm{N}=35)$ & ICIs $(\mathrm{N}=31)$ & $p$-Value \\
\hline Median age, y (range) & $74.5(70-91)$ & $75(70-85)$ & $74(70-91)$ & 0.92 \\
\hline Gender, Male/Female & $46 / 20$ & $24 / 11$ & $22 / 9$ & 0.83 \\
\hline Smoking status, yes/no & $48 / 18$ & $26 / 9$ & $22 / 9$ & 0.76 \\
\hline Stage, III/IV & $17 / 49$ & $12 / 23$ & $5 / 26$ & 0.08 \\
\hline ECOG PS, 0 or $1 / 2$ & $45 / 21$ & $28 / 7$ & $17 / 14$ & 0.027 \\
\hline Histology, non $\mathrm{Sq} / \mathrm{Sq}$ & $44 / 22$ & $25 / 10$ & $19 / 12$ & 0.38 \\
\hline \multicolumn{5}{|l|}{ PD-L1 expression (22C3) } \\
\hline High & 7 & 1 & 6 & \\
\hline Low & 6 & 0 & 6 & \\
\hline No & 4 & 1 & 3 & \\
\hline $\mathrm{NE}$ & 49 & 33 & 16 & \\
\hline \multicolumn{5}{|l|}{$E G F R$ mutation } \\
\hline Positive & 9 & 4 & 5 & \\
\hline Negative or unknown & 57 & 31 & 26 & 0.57 \\
\hline \multicolumn{5}{|l|}{ Prior EGFR-TKIs, } \\
\hline Yes/no & $9 / 57$ & $4 / 31$ & $5 / 26$ & 0.57 \\
\hline \multicolumn{5}{|c|}{ Immune checkpoint inhibitor } \\
\hline Nivolumab & & & 23 & \\
\hline Pembrolizumab & & & 8 & \\
\hline \multicolumn{5}{|l|}{ Cytotoxic chemotherapy } \\
\hline Docetaxel & & 13 & & \\
\hline Pemetrexed & & 9 & & \\
\hline TS-1 & & 6 & & \\
\hline Nab PTX & & 4 & & \\
\hline Others & & 3 & & \\
\hline \multicolumn{5}{|l|}{ Charlson risk index } \\
\hline Low: score 0 & 35 & 18 & 17 & \\
\hline Score $\geq 1$ & 31 & 17 & 14 & 0.78 \\
\hline Medium: 1-2 & 25 & 14 & 11 & \\
\hline High: $3-4$ & 5 & 3 & 2 & \\
\hline Very high: $\geq 5$ & 1 & 0 & 1 & \\
\hline
\end{tabular}

ICIs: Immune checkpoint inhibitors; EGFR-TKIs: epidermal growth factor receptor; TKIs: tyrosine kinase inhibitors; NE: not evaluate; ECOG PS: eastern cooperative oncology group performance status; Sq: Squamous; PD-L1: programmed cell death protein ligand 1; nab PTX: nab paclitaxel.

free survival (PFS) and OS were defined as the interval from the start of second cytotoxic chemotherapy or the initiation of ICIs to disease progression or death from any cause, respectively. The cutoff date was August 30, 2019. PFS and OS were analyzed using the Kaplan-Meier method and compared using the log-rank test. The likelihood ratio test for interactions was used to assess treatment effects in respect to baseline characteristics. Furthermore, multivariate analysis was performed using the Cox proportional hazards model.

A $p$-value $<0.05$ was considered to indicate a statistically significant difference. All analyses were performed using JMP version 8.0 (SAS Institute, Cary, NC, USA).

The study protocol was approved by the institutional review board of the Yokohama Municipal Citizen's Hospital. A written informed consent from patients was not required because individual patients were not identified in this retrospective study.

\section{Results}

Patient characteristics. In our institute, 364 patients $\geq 70$ years old were diagnosed with NSCLC based on histology or cytology, between January 2011 and March 2018. We present the diagram of patient selection in Figure 1. Seventytwo patients were treated with two or more chemotherapy regimens. Excluding 6 patients treated with ICIs as fourth or later lines, we evaluated 66 patients. There were 35 patients in the No ICIs group and 31 patients in the ICIs group. In the ICIs group, 23 patients were treated with nivolumab and 8 patients with pembrolizumab.

Patient characteristics are shown in Table I. The median ages were 75 years (range $=70-85$ years) in the No ICIs group and 74 years (range=70-91 years) in the ICIs group. No significant differences in age, sex, smoking status, stage, histology, and prior EGFR-TKIs were found between the two groups. Fewer patients in the No ICIs group than in the ICIs group had a poor performance status (PS). In the No ICIs group, docetaxel was the most commonly administered regimen; the second most frequent regimen was pemetrexed. According to analyzing the Charlson Risk Index (CRI), 31 patients $(47 \%)$ had $s c o r e \geq 1$. However, there were no 
364 patients with $\geq 70$ years old and non small cell lung cancer

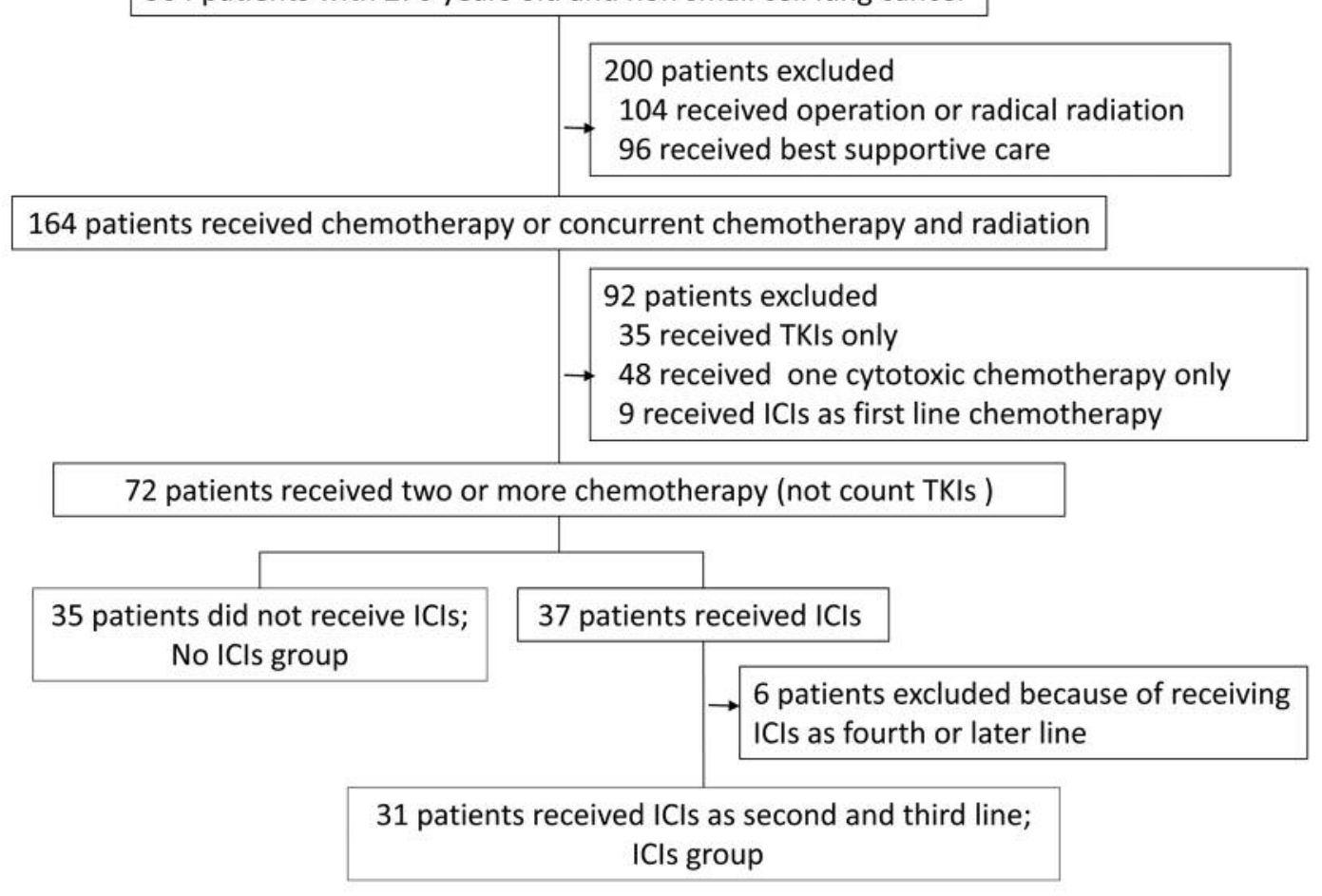

Figure 1. The diagram of patient selection. ICIs: Immune checkpoint inhibitors; TKIs: tyrosine kinase inhibitors.

significant differences in CRI between the ICIs group and the No ICIs group (Table I).

Progression-free survival and overall survival. The median PFS in the No ICIs and ICIs groups were 3.8 months and 2.4 months, respectively $(p=0.98)$ (Figure $2 \mathrm{~A})$, and the corresponding median OS were 8.9 months and 9.6 months, respectively ( $p=0.67$ ) (Figure $2 \mathrm{~B}$ ). The median OS in patients with squamous cell carcinoma was significantly different between the two groups [9.7 months in the ICIs group versus 4.7 months in the No ICIs group ( $p=0.027)$ ] (Figure 3A). In contrast, the median OS in patients with non squamous cell carcinoma was not different between the ICIs group and the No ICIs group (Figure 3B). The likelihood ratio test regarding the interaction between baseline characteristics and treatment effect is shown in Figure 4. ICIs were only effective in squamous cell carcinoma patients.

Multivariate analysis. In the multivariate analysis, treatment with ICIs $(\mathrm{HR}=0.54,95 \% \mathrm{CI}=0.30-0.98, p=0.044)$, good Eastern Cooperative Oncology Group (ECOG) PS (HR=0.30, $95 \% \mathrm{CI}=0.16-0.57, p=0.0003)$, and histology other than squamous cell carcinoma $(\mathrm{HR}=0.41,95 \% \mathrm{CI}=0.19-0.83$, $p=0.014$ ) were significantly favorable factors for OS (Table II).
Adverse events in the ICIs group. In the ICIs group, grade $\geq 3$ AEs occurred in 6 patients $(19 \%)$. The grade $\geq 3$ AEs were skin rash [2(6\%) patients], elevation of aspartate aminotransferase [1 (3\%) patient], hyperglycemia [1 (3\%) patient], and pneumonitis [2 $(6 \%)$ patients]. There was no treatment-related death.

\section{Discussion}

Anti-PD-1 inhibitors are an established standard therapy for patients with previously treated advanced NSCLC. However, the effect of these ICIs in older patients has not been adequately assessed. Here, we investigated the effect of ICIs in older ( $\geq 70$ years old) NSCLC patients, previously treated with chemotherapies, comparing their outcomes to those of patients treated with cytotoxic chemotherapy only. Our study demonstrated that the median OS in patients with squamous cell carcinoma was significantly different between the two groups [9.7 months in the ICIs group versus 4.7 months in the No ICIs group $(p=0.027)]$. The likelihood ratio test for interactions showed that the HR for squamous cell carcinoma patients was 0.33 (95\% CI=0.11-0.90). The Checkmate 017 trial showed that OS was significantly better with nivolumab than with docetaxel among patients with advanced, previously treated squamous cell carcinoma (3). In that trial, 
A

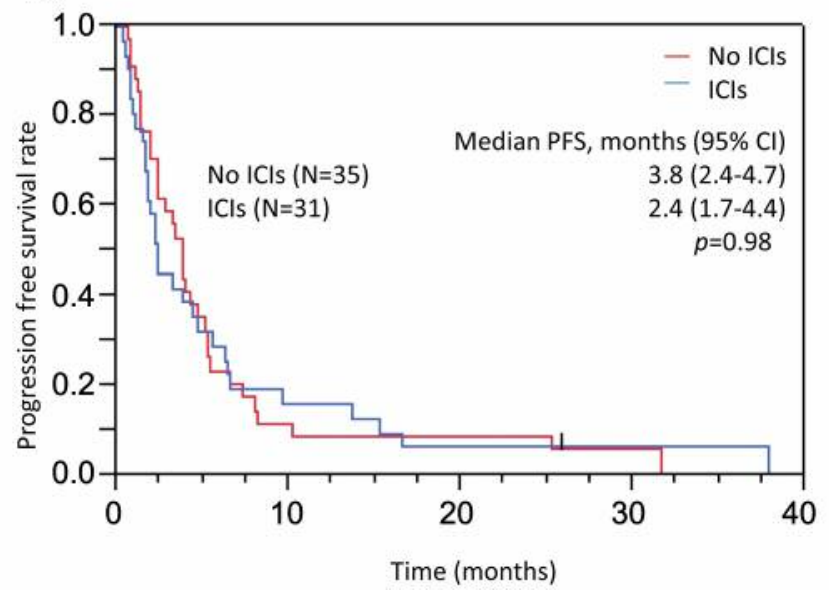

B

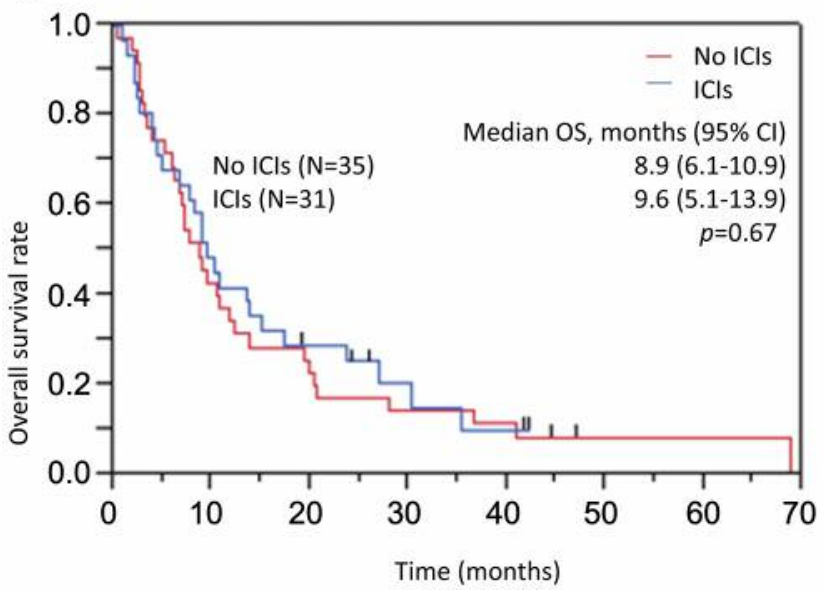

Figure 2. Kaplan-Meier curves of (A) progression-free survival and $(B)$ overall survival in patients aged $\geq 70$ who were or were not treated with ICIs. ICIs: Immune checkpoint inhibitors; PFS: progression-free survival; OS: overall survival; CI: confidence interval.

A

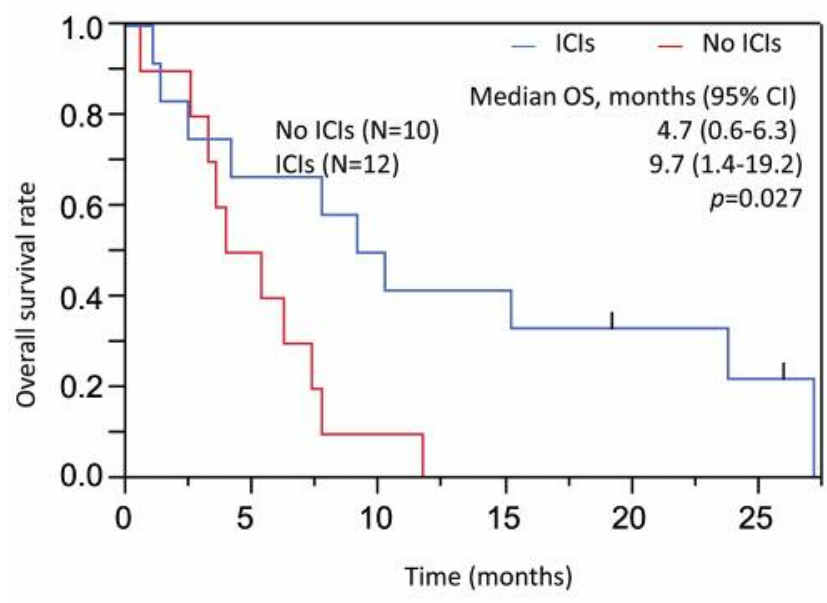

B

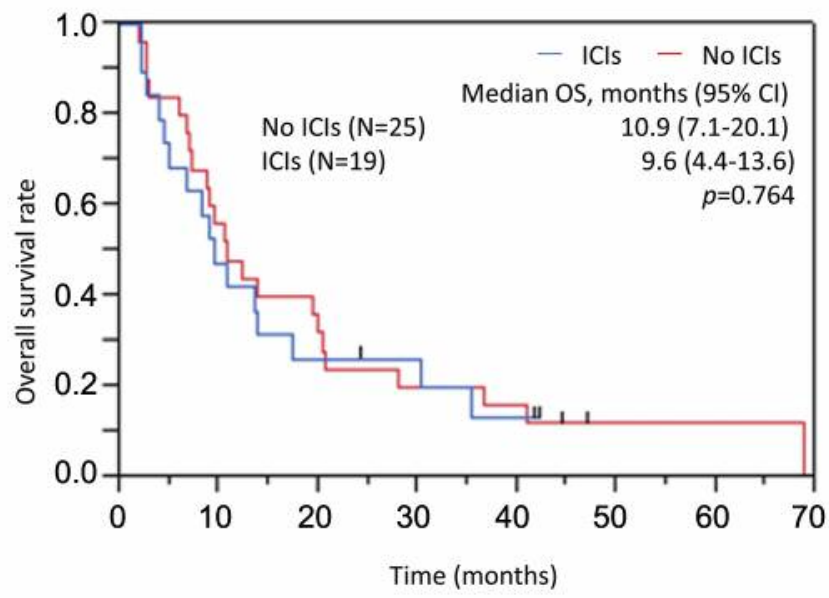

Figure 3. Kaplan-Meier curves of overall survival in (A) squamous cell carcinoma patients and (B) non-squamous cell carcinoma patients aged $\geq 70$ who were or were not treated with ICIs. ICIs: Immune checkpoint inhibitors; OS: overall survival; CI: confidence interval.

the median overall survival was 9.2 months $(95 \% \mathrm{CI}=7.3$ 13.3 months) with nivolumab versus 6.0 months (95\% CI=5.1-7.3 months) with docetaxel. The median OS in our study was comparable to that in Checkmate 017 . The Checkmate 171 trial evaluated the safety and efficacy of nivolumab monotherapy in advanced squamous cell carcinoma patients with progressive disease after $\geq 1$ systemic treatment (9). In subgroup analysis based on age $\geq 70$, the median OS was 11.2 months, and the estimated 6 -month OS was $66 \%$. These findings support the same potential efficacy of ICIs for older patients with squamous cell carcinoma.
In the total population, we could not find any difference in PFS or OS between the ICIs group and the No ICIs group. This could be because the proportion of patients with ECOG PS 2 was higher in the ICIs group than in the No ICIs group ( $45 \%$ versus $20 \%, p=0.027$ ). In the multivariate analysis, treatment with ICIs was a favorable prognostic factor for OS, as were good ECOG PS and histology other than squamous cell carcinoma. These results suggest that ICIs might be an effective treatment for older patients with previously treated NSCLC, as they are younger patients. A meta-analysis showed that PD-1 inhibitors were associated with significantly longer OS in both younger ( $<65$ years old) and 


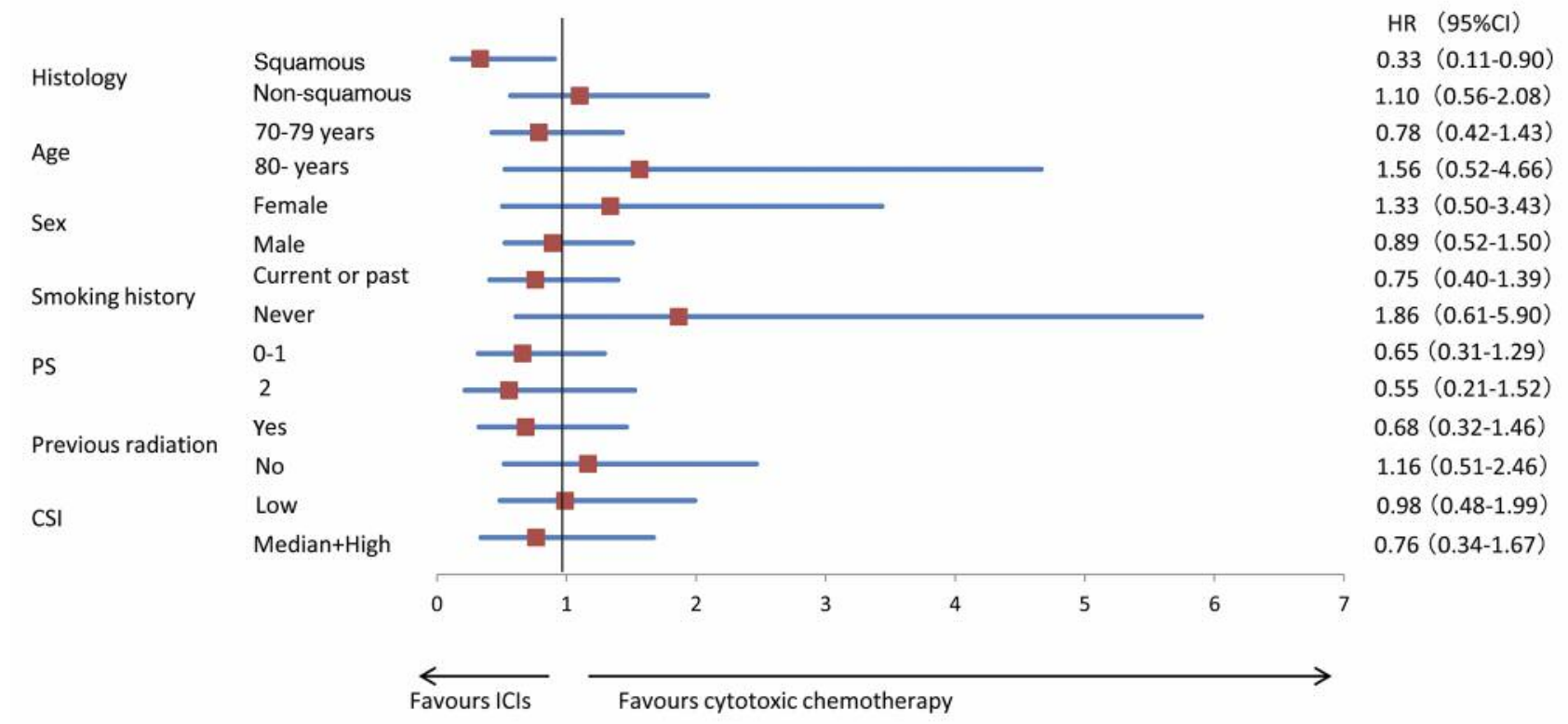

Figure 4. The likelihood ratio test for interactions was used to assess the treatment effects based on different baseline characteristics. ICIs: Immune checkpoint inhibitors; PS: performance status; HR: hazard ratio; CI: confidence interval; CSI: Charlson Risk Index.

older ( $\geq 65$ years old) groups compared with chemotherapy (6). However, in this meta-analysis, among NSCLC patients aged over 75, no prolonged OS was observed compared with chemotherapy. A previous study showed that $\mathrm{T}$ cells undergo senescent changes in patients older than 70 years (10). Moreover, aging is associated with a decrease in CD8+ T cells, which are influenced by PD-1 signaling (11). Because of the decreasing function of the immune system with age, ICIs may have different effects in patients of different ages. On the other hand, our study showed an effect of ICIs in the older population as in seen a few previous reports. Therefore, we need to clarify the relationship between the immune system and the effects of ICIs in older patients.

Treatment-related AEs of grade 3 or 4 were reported in $19 \%$ of patients in the ICIs group. The incidence of treatment-related AEs of grade 3 or 4 in previous trials of nivolumab and pembrolizumab for all patients with previously treated NSCLC was about 7-10\% (1-3). Our study showed a higher incidence of AEs of grade 3 or 4 . Pneumonitis was reported in 2 patients $(6 \%)$, which is rather high compared to historical data of nivolumab and pembrolizumab (1-3). However, no treatment-related death occurred. As deteriorating organ function is more common among older patients than among younger patients, it is necessary to use ICIs with caution, although this is also a risk with cytotoxic agents.

Certain limitations should be considered in interpreting this retrospective analysis. First, this study relied on a
Table II. Multivariate analysis.

\begin{tabular}{lllll}
\hline & & Risk & 95\%CI & $p$-Value \\
\hline Treatment with ICIs & Yes/No & 0.54 & $0.30-0.98$ & 0.044 \\
Age & $<75 / \geq 75$ & 1.13 & $0.67-1.90$ & 0.66 \\
Gender & Female/Male & 0.83 & $0.43-1.60$ & 0.58 \\
Smoking status & Yes/No & 0.76 & $0.37-1.57$ & 0.45 \\
ECOG PS & 0 or $1 / 2$ & 0.30 & $0.16-0.57$ & 0.0003 \\
Histology & No Sq/Sq & 0.41 & $0.19-0.83$ & 0.014 \\
\hline
\end{tabular}

ICIs: Immune checkpoint inhibitors; ECOG PS: eastern cooperative oncology group performance status; Sq: squamous cell carcinoma; CI: confidence interval.

retrospective chart review of patients treated in a practical clinical setting. Therefore, the possibility of some bias in the physician's decision cannot be denied. Second, because of the change in standard care, there were differences in the periods between the ICIs and No ICIs groups. We analyzed patients between January 2011 and March 2018; non-ICI treatment was more frequently administered at the beginning of the analysis period, and ICI treatment was more recently frequent.

In conclusion, our study demonstrated that ICIs may be as effective for older patients as they are for younger patients, especially for those with squamous cell carcinoma. As the population of older patients continues to expand, it will 
become increasingly necessary to identify the effect of novel chemotherapeutic agents on older patients in a real-world setting.

\section{Conflicts of Interest}

All Authors state that they have no conflicts of interest associated with this manuscript.

\section{Authors' Contributions}

Study concept and design: Yukiko Nakamura; Drafting of the manuscript: Yukiko Nakamura; Critical revision of the manuscript: Kazuhito Miyazaki, Naoto Aiko, Yuki Misumi, Yoko Agemi, Yuri Taniguchi, Mari Ishi, Tsuneo Shimokawa; Study supervision: Hiroaki Okamoto and Takeharu Yamanaka.

\section{Acknowledgements}

The Authors are grateful to Editage (www. Editage. jp) for English language editing.

\section{References}

1 Herbst RS, Baas P, Kim DW, Felip E, Pérez-Gracia JL, Han JY, Molina J, Kim JH, Arvis CD, Ahn MJ, Majem M, Fidler MJ, de Castro G Jr., Garrido M, Lubiniecki GM, Shentu Y, Im E, Dolled-Filhart $\mathrm{M}$ and Garon EB: Pembrolizumab versus docetaxel for previously treated, PD-L1-positive, advanced nonsmall-cell lung cancer (KEYNOTE-010): a randomised controlled trial. Lancet 387(10027): 1540-1550, 2016. PMID: 26712084. DOI: $10.1016 /$ S0140-6736(15)01281-7

2 Borghaei H, Paz-Ares L, Horn L, Spigel DR, Steins M, Ready NE, Chow LQ, Vokes EE, Felip E, Holgado E, Barlesi F, Kohlhäufl M, Arrieta O, Burgio MA, Fayette J, Lena H, Poddubskaya E, Gerber DE, Gettinger SN, Rudin CM, Rizvi N, Crinò L, Blumenschein GR Jr., Antonia SJ, Dorange C, Harbison CT, Graf Finckenstein F and Brahmer JR: Nivolumab versus docetaxel in advanced nonsquamous non-small-cell lung cancer. N Engl J Med 373(17): 1627-1639, 2015. PMID: 26412456. DOI: $10.1056 /$ NEJMoa1507643

3 Brahmer J, Reckamp KL, Baas P, Crinò L, Eberhardt WE, Poddubskaya E, Antonia S, Pluzanski A, Vokes EE, Holgado E, Waterhouse D, Ready N, Gainor J, Arén Frontera O, Havel L, Steins M, Garassino MC, Aerts JG, Domine M, Paz-Ares L, Reck M, Baudelet C, Harbison CT, Lestini B and Spigel DR: Nivolumab versus docetaxel in advanced squamous-cell nonsmall-cell lung cancer. N Engl J Med 373(2): 123-135, 2015. PMID: 26028407. DOI: 10.1056/NEJMoa1504627

4 Torre LA, Siegel RL and Jemal A: Lung cancer statistics. Adv Exp Med Biol 893: 1-19, 2016. PMID: 26667336. DOI: 10.1007/978-3-319-24223-1_1
5 Khozin S, Abernethy AP, Nussbaum NC, Zhi J, Curtis MD, Tucker M, Lee SE, Light DE, Gossai A, Sorg RA, Torres AZ, Patel P, Blumenthal GM and Pazdur R: Characteristics of realworld metastatic non-small cell lung cancer patients treated with nivolumab and pembrolizumab during the year following approval. Oncologist 23(3): 328-336, 2018. PMID: 29317551. DOI: $10.1634 /$ theoncologist.2017-0353

6 Wu Y, Ju Q, Qian B, Zhang F and Shi H: The effectiveness of PD-1 inhibitors in non-small cell lung cancer (NSCLC) patients of different ages. Oncotarget 9(8): 7942-7948, 2018. PMID: 29487704. DOI: $10.18632 /$ oncotarget.23678

7 Spigel DR, McCleod M, Jotte RM, Einhorn L, Horn L, Waterhouse DM, Creelan B, Babu S, Leighl NB, Chandler JC, Couture F, Keogh G, Goss G, Daniel DB, Garon EB, Schwartzberg LS, Sen R, Korytowsky B, Li A, Aanur N and Hussein MA: Safety, efficacy, and patient-reported health-related quality of life and symptom buren with nivolumab in patients with advanced non-small cell lung cancer, including patients aged 70 years or older or with poor performance status (CheckMate 153). J Thorac Oncol 14(9): 1628-1639, 2019. PMID: 31121324. DOI: 10.1016/j.jtho.2019.05.010

8 Grossi F, Crinò L, Logroscino A, Canova S, Delmonte A, Melotti B, Proto C, Gelibter A, Cappuzzo F, Turci D, Gamucci T, Antonelli P, Marchetti P, Santoro A, Giusti S, Di Costanzo F, Giustini L, Del Conte A, Livi L, Giannarelli D and de Marinis F: Use of nivolumab in elderly patients with advanced squamous non-small-cell lung cancer: results from the Italian cohort of expanded access programme. Eur J Cancer 100: 126-134, 2018. DOI: $10.1016 /$ j.ejca.2018.05.015

9 Popat S, Arduzzoni A, Ciuleanu T, Cobo Dols M, Laktionov K, Szilasi M, Califano R, Carcereny Costa E, Griffiths R, Paz-Ares L, Szczylik C, Corral J, Isla D, Jassem J, Appel W, van Meerbeeck J, Wolf J, Molife LR and Felip Font E: Nivolumab in previously treated patients with metastatic squamous NSCLC: Results of a European single-arm, phase 2 trial (CheckMate 171) including patients aged $\geq 70$ years and with poor performance status. Ann Oncol 28: 463, 2017. DOI: 10.1093/annonc/mdx380.006

10 Farber DL, Yudanin NA and Restifo NP: Human memory T cells: generation, compartmentalization and homeostasis. Nat Rev Immunol 14(1): 24-35, 2014. PMID: 24336101. DOI: $10.1038 /$ nri3567

11 Shao H, Ou Y, Wang T, Shen H, Wu F, Zhang W, Tao C, Yuan $\mathrm{Y}$, Bo $\mathrm{H}$, Wang $\mathrm{H}$ and Huang S: Differences in TCR-V $\beta$ repertoire and effector phenotype between tumor infiltrating lymphocytes and peripheral blood lymphocytes increase with age. PLoS One 9(7): e102327, 2014. PMID: 25019226. DOI: 10.1371 /journal.pone. 0102327 